\title{
INDECOMPOSABILITY OF IDEALS IN GROUP RINGS
}

\author{
M. M. PARMENTER ${ }^{1}$
}

\begin{abstract}
Let $H$ be a subgroup of $G$ and let $I$ be the (two-sided) ideal of $\mathbf{Z} G$ generated by $\omega(\mathbf{Z} H)$. In this note, we show that $I$ is indecomposable as an ideal in $\mathbf{Z} G$. This extends a result of Linnell [1] and simplifies his argument somewhat.
\end{abstract}

If $H$ is a group, we will denote the augmentation ideal of the integral group ring $\mathbf{Z} H$ by $\omega(\mathbf{Z} H)$. In this very brief note, we prove the following result .

THEOREM. Let $H$ be a subgroup of $G$ and let $I$ be the (two-sided) ideal of $\mathbf{Z} G$ generated by $\omega(\mathbf{Z H})$. Then $I$ is indecomposable as an ideal in $\mathbf{Z} G$.

The case where $H$ is a normal subgroup of $G$ was recently proved by Linnell [1]. Our argument is somewhat simpler and, of course, extends to arbitrary subgroups.

ProOF OF THEOREM. Suppose $I=P \oplus Q$ is a decomposition as an ideal of $\mathbf{Z} G$.

First consider the case where $H$ is a torsion subgroup of $G$ and let $h \in H$. Then $h-1=p+q$ where $p \in P, q \in Q$ and $p q=q p=0$.

Hence, for some $k,(1+p+q)^{k}=h^{k}=1$.

Thus, $k(p+q)+\left(\begin{array}{c}k \\ 2\end{array}\right)\left(p^{2}+q^{2}\right)+\cdots+\left(p^{k}+q^{k}\right)=0$. Since $P \cap Q=0$, we conclude that $(1+p)^{k}=(1+q)^{k}=1$. Therefore, $1+p$ and $1+q$ are units of finite order in $\mathbf{Z} G$. Since $(1+p)+(1+q)=1+h$, either $1+p$ or $1+q$ must have a nonzero identity coefficient. By [3, Corollary 2.1.3] and the fact that $p$ and $q$ are contained in $\omega(\mathbf{Z} G)$, we conclude that either $1+p=1$ or $1+q=1$. Hence $h-1 \in P$ or $h-1 \in Q$. Because $\left(h_{1}-1\right)\left(h_{2}-1\right) \neq 0$ if $h_{1} \neq h_{2}$ and $h_{1}, h_{2} \neq 1$, we conclude that $I=P$ or $I=Q$, and we are done.

When $H$ is not torsion, we copy part of the argument in [1]. Let $F$ be the torsion subgroup of the finite conjugate subgroup of $G$ and let $\pi: \mathbf{Z} G \rightarrow \mathbf{Z} F$ be the natural projection. Since $H \nsubseteq F$, we have $\pi(I)=\mathrm{Z} F$. By [2, Theorems 4.2.12 and 4.3.16], we conclude that $\mathbf{Z} F=\pi(I)=\pi(P) \oplus \pi(Q)$ with $\pi(P) \neq 0 \neq \pi(Q)$. However, this contradicts the fact that $\mathbf{Z} F$ is indecomposable [2].

\section{REFERENCES}

1. P. A. Linnell, Indecomposability of the augmentation ideal as a two-sided ideal, J. Algebra 82 (1983), 328-330.

2. D. S. Passman, The algebraic structure of group rings, Interscience, New York, 1977.

3. S. K. Sehgal, Topics in group rings, Dekker, New York, 1978.

Department of MAthematics, Memorial University of NeWfoundland, ST. JOHN'S, NEWFOUNDLAND A1B 3X7, CANADA

Received by the editors October 19, 1983.

1980 Mathematics Subject Classification. Primary 16A26.

${ }^{1}$ This work supported in part by NSERC Grant A-8775. 